\title{
Passive Wireless SAW Sensors for IVHM
}

\author{
William C. Wilson, Daniel F. Perey \\ Nondestructive Evaluation Branch \\ NASA Langley Research Center \\ Hampton VA, 23681, USA
}

\author{
Gary M. Atkinson \\ Electrical and Computer Engineering \\ Virginia Commonwealth University \\ Richmond, VA, 23284, USA \\ gmatkins@vcu.edu
}

\author{
Rebecca O. Barclay \\ Department of English \\ Christopher Newport University, \\ Newport News, VA, USA
}

\begin{abstract}
NASA aeronautical programs require integrated vehicle health monitoring (IVHM) to ensure the safety of the crew and the vehicles. Future IVHM sensors need to be small, light weight, inexpensive, and wireless. Surface acoustic wave (SAW) technology meets all of these constraints. In addition it operates in harsh environments and over wide temperature ranges, and it is inherently radiation hardened. This paper presents a survey of research opportunities for universities and industry to develop new sensors that address anticipated IVHM needs for aerospace vehicles. Potential applications of passive wireless SAW sensors from ground testing to high altitude aircraft operations are presented, along with some of the challenges and issues of the technology.
\end{abstract}

\section{INTRODUCTION}

Aerospace vehicles require integrated vehicle health monitoring (IVHM) to ensure the safety of the crew and the vehicle. Frequently, constraints such as cost, mass, volume, and power, prevent the addition of IVHM instrumentation onto aircraft. One solution to these constraints is to eliminate wiring and wiring harnesses to reduce the mass of vehicle health monitoring systems [1]. Also, using wireless instead of wired sensors for IVHM applications avoids expensive redesigns to route cables and the costs of performing safety recertifications [2]. The Decadal Survey of Civil Aeronautics [3] identifies IVHM as the top NASA and national priority within the area of materials and structures. The survey also indicated that "self-powered, wireless microelectromechanical sensors" warrant attention over the next decade. Current wireless sensor systems have low data rates and require batteries. The environment of aerospace vehicles is typically harsh, with temperature extremes ranging from cryogenic to very high temperatures. For example, the hypersonic X-43 vehicle will require high temperature sensors mounted on the structure, as well as cryogenic sensors for monitoring fuel tanks. Sensors are typically located in internal structures with limited access, making the periodic changing of batteries costly and time consuming. Furthermore, batteries do not work well in either temperature extreme. In contrast to current wireless systems, passive wireless surface acoustic wave (SAW) sensors operate across a large temperature range and do not require batteries. As a result, NASA is investigating the

This work is funded by NASA Integrated Vehicle Heath Management (IVHM) Project which is part of the Aviation Safety Program under the Aeronautics Research Mission Directorate (ARMD) use of SAW technology for aerospace applications. From ground tests to the operation of high altitude long duration aircraft, many applications could benefit from small, passive, wireless sensors.

\section{GENERAL REQUIREMENTS}

The sensing needs for IVHM of aircraft span the range from low to extremely high data sampling rates. Simple measurements, such as temperature, pressure, strain, and acceleration, fall in the category of low data rate devices. These rates may be as low as one sample per hour for slowly changing parameters. IVHM techniques, such as imaging, eddy current, and terahertz waves, all require moderate data rates.

However, acoustic emission, thermography, and ultrasonics require higher rates, on the order of mega samples per second. Current RFID technology cannot transmit at these rates; therefore, SAW technology is being investigated.

Aircraft sensing equipment must also operate in harsh environments that include temperatures ranging from cryogenic to extremely high temperatures. Often, the temperature extremes preclude the use of batteries for sensor applications. Besides temperature extremes, issues of vibration, humidity, and even ionizing radiation must be addressed.

In addition to the other challenges, the RF environment can also pose a challenge when wireless RF sensors are placed within enclosed metallic structures, such as the interior of wings. To alleviate some of the RF issues, SAW devices can use complex RF communication techniques. SAW devices have been modulated in many ways, such as frequency modulated continuous wave [4] and orthogonal frequency coding [5]. These techniques, along with other spread spectrum technologies, reduce some of the problems caused by multipath reflections of RF signals.

Passive wireless sensors based upon SAW technology offer the potential to meet all these requirements. The use of SAW devices as ID tags is not new [6,7]. Also, a variety of wireless sensors have already been created. Sensors for temperature, humidity, pressure, torque, current, and 
magnetoresistivity, have already been developed [8, 9]. SAW technology is ready to be applied towards the creation of passive wireless IVHM sensors for aircraft applications.

\section{SAW DEVICES DEVELOPED BY NASA}

The field of SAW devices was initiated when White and Voltmer invented the interdigital transducer (IDT) in 1965. At NASA Langley Research Center, an imaging device that used surface-acoustic-wave (SAW) charge transfer for image readout was developed in 1985 [10]. In the device, traveling surface acoustic waves created moving electric fields. The fields generated potential wells that carried along stored charges. When light struck the device, charges were injected into the wells by photoelectric conversion. The spatial resolution of the image was varied electronically by modifying the frequency of applied signal.

In 1992, the signal processing capabilities of SAW devices were exploited to create down chirp bandpass filters for satellite communications [11]. The SAW reflective array compressor chirp filter has an operating frequency that is centered at $300 \mathrm{MHz}$. The SAW filters were chosen because their roughly rectangular-shaped bandpass is ideal for rejecting spurious signals and interference.

NASA partnered with White in 1997 to investigate atmospheric sensing using SAW and flexural plate wave (FPW) devices [12]. The devices were designed as chemical detectors to measure the amount of nitrous oxide present in the atmosphere.

SAW technology enabled the development of a micro hygrometer for in situ meteorology [13]. The instrument is faster than chilled mirror hygrometers, and more reliable than humidity sensors. Tropospheric humidity was mapped using the micro hygrometer on a small radiosonde balloon. A micro hygrometer was also flown on a NASA DC-8 as part of the convection and moisture experiment (CAMEX), to provide high resolution in situ humidity measurements. In 1999, the device was flown on NASA's Helios unpiloted aircraft.

NASA continues to look for reliable, sensitive, robust, low power sensors for chemical, mineral and biological detection. In 2003 SAW and bulk acoustic wave (BAW) sensors were investigated for a variety of in situ measurements [11]. The wireless capabilities of BAWs and SAWs were also explored.

A team at the NASA Johnson Space Center (JSC) is testing SAW Radio Frequency Identification (RFID) technology to track assets aboard the International Space Station (ISS) [14]. Because, standard IC-based RFID tags cannot be read reliably enough for this application, SAW RFID devices are being implemented. SAW RFID tags have better signal penetration in and around liquids and metals, and have more robust anti-collision capabilities; therefore, they are being implemented to track consumables such as meals on board the ISS.

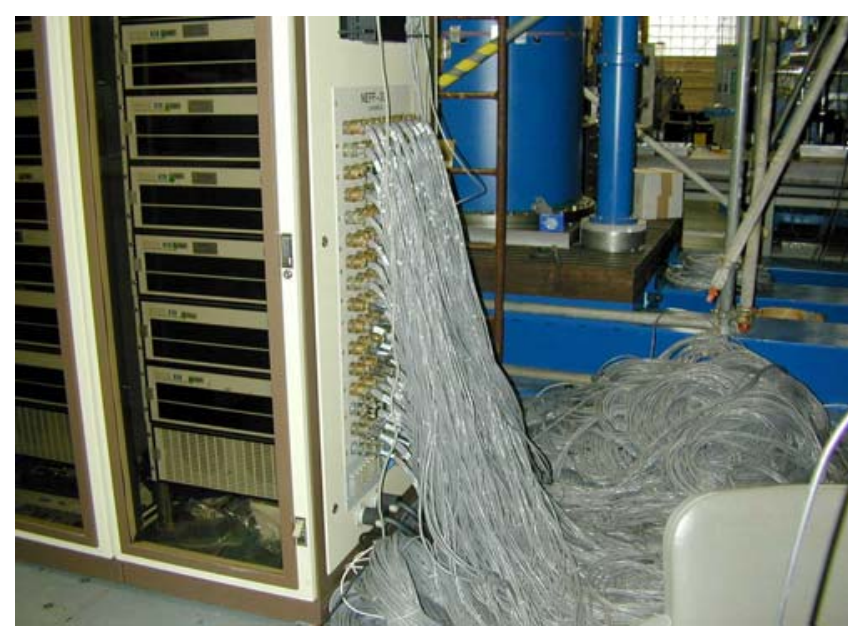

Figure 1. Photograph of the cabling required to connect 466 foil strain gauges to a wing box test article.

\section{GROUnd Testing Applications}

NASA aeronautical researchers perform tests on components and systems on the ground in conjunction with flight testing. These tests require the placement of large numbers of sensors on a test article. To date, almost none of the sensors is connected wirelessly. For example, a stitched/resin film infused graphite-epoxy wing box was tested using 446 strain gauge sensors connected to the test article using standard cabling [15]. The time and cost to install and troubleshoot the large numbers of cables can be eliminated. Figure 1 shows a photograph of the cables required to connect the 446 strain gages to the test article.

In addition to aeronautics, other disciplines could benefit from passive wireless sensors when NASA performs ground tests. At Kennedy Space Center (KSC), wireless sensor networks have been developed for monitoring cryogenic lines and for centering and aligning the external tank [16]. Ongoing work in the Transducers group at KSC integrates wireless communications with sensors and transducers. At Langley Research Center, researchers have developed and tested a wireless fluid level system that works in such harsh environments as immersion in liquid nitrogen [17]. The device can work in a variety of harsh environments while detecting the level of numerous "fluids" such as liquid nitrogen, transmission fluid, sugar, and even ground corn. While the devices just mentioned do not employ passive SAW technology, they do demonstrate the current trend towards wireless sensing for ground testing.

NASA often uses thermo-vacuum chambers to test aircraft and spacecraft systems and components. In this scenario, there is a need to reduce the number of wires that must penetrate the chamber. Requirements that limit the amount of out gassing and temperature extremes can rule out the use of batteries for this application. Wireless passive sensors such as SAW devices, however, could reduce the number of wires penetrating the chamber without introducing the need for batteries. 
A current example of SAW devices being investigated is the Passive Wireless SAW Sensor Tag project funded by the NASA Engineering Safety Center (NESC), and run by Johnson Space Center. This project is developing a system that would use passive RF SAW tags to eliminate the wires between high data-rate and high-impedance sensors. The initial application of the system is acceleration and acoustic emission measurements during acoustic vibration ground testing of space structures, but the system could be used for many types of ground tests.

\section{AIRCRAFT PROPULSION APPLICATIONS}

Active wireless sensor systems have been developed for monitoring the health of aircraft engines for commercial, military, and NASA aircraft [18-20], but all of these systems require batteries. NASA would prefer future sensors to be passive. The Army, Air Force, and NASA require high temperature propulsion sensors that can operate in environments of up to $1538^{\circ} \mathrm{C}$ around the engine and inside the gas path [21]. Both wiring and batteries become an issue in these applications; therefore, high temperature-resistant, passive wireless sensors, like the passive engine-bearing sensor [22], are needed.

High temperature materials are currently being researched for SAW sensor applications. Aluminum nitride is being investigated for high temperature $\left(800^{\circ} \mathrm{C}\right)$ operation of temperature compensating sensors [23]. Gallium phosphate $(\mathrm{GaPO})$ has been used as a substrate in the development of a temperature sensor [24]. The sensor is wireless, operates at $433 \mathrm{MHz}$, and withstands temperatures of $600^{\circ} \mathrm{C}$ for 192 hours. Exotic materials such as langatite and langatate have been characterized from $-100^{\circ} \mathrm{C}$ to $200^{\circ} \mathrm{C}$ [25]. These new materials may enable SAW sensors to operate at greater than $1000^{\circ} \mathrm{C}$.

\section{AirCRAFt StRUCTURAL APPLiCATIONS}

NASA envisions adding IVHM sensors to existing aircraft; however, installing wiring for the sensors adds cost and weight to the aircraft. Also, wires are prone to damage such as nicks, breaks, degradation due to wear, excessive heating, and arcing. Wiring problems have led to major aircraft accidents and delays of space vehicle launches [26]. In contrast, wireless systems present a desirable option for retrofitting sensors onto existing aircraft for structural health monitoring.

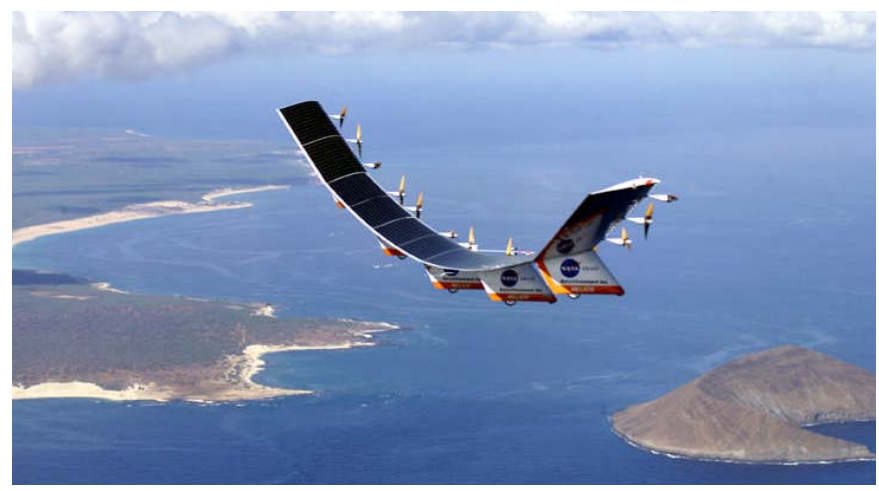

Figure 2. Helios (HP01), one of NASA's ultra-long duration solar aircraft.
NASA has been investigating the use of ultra-long duration solar powered aircraft (Fig. 2), which would fly missions for weeks or months. These aircraft need real-time dihedral sensors to detect the exact shape of the wing as it bends [27]. For this application the sensors must be extremely lightweight and low power. Passive wireless sensors would provide these characteristics and eliminate wiring weight as well.

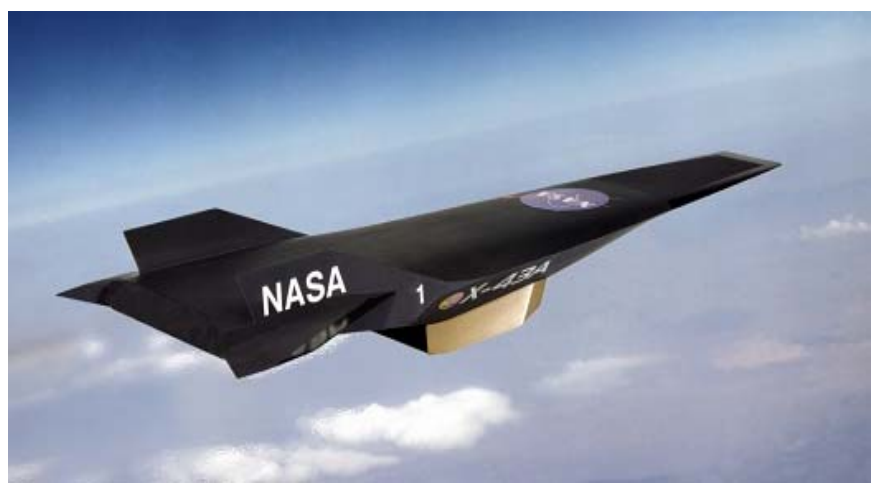

Figure 3. Artist Conception of X-43A Hypersonic Experimental Vehicle.

High speeds mean high temperatures from skin friction heating. Sensors for hypersonic (greater than mach 5) aircraft and aerospace vehicles may experience aerodynamic heating above $1000^{\circ} \mathrm{C}$. NASA's HyperX X-43 (Fig. 3) aircraft will fly at mach 10 and therefore will require sensors that must be able to withstand temperatures up to $1282^{\circ} \mathrm{C}$ [28]. Thus, hypersonic vehicles will need high temperature wireless sensors like those needed for propulsion applications.

The Space Shuttle, HyperX, and Helios all need high temperature chemical sensors that can detect hydrogen [29]. A SAW hydrogen sensor based on a langasite substrate and utilizing palladium as the sensing medium has been shown to operate at $250^{\circ} \mathrm{C}$ [30]. Since SAW technology can be used for hydrogen sensing, all that is needed is the addition of passive wireless capability. Other chemical sensing is needed as well. The integrity of wires on board aerospace vehicles could be determined by monitoring the effluents given off by the wire's insulation [31]. The effluents are generated during aging, over-currents, arcing, and high temperature conditions. SAW chemical sensors could be used to detect effluents and thereby give an indication of wire integrity.

At the other temperature extreme, cryogenic liquids are often found in aerospace vehicles. The external structures may experience extremely low temperatures as well. For these applications, sensors that can operate or withstand low temperatures are required. Cryogenic liquid operation of SAW devices has been demonstrated [32].

High-altitude-sensing applications require sensors that exhibit tolerance to ionizing radiation. Tests conducted on SAW devices demonstrated an inherent radiation tolerance up to 10 Mrad [33]. The constant size reduction of commercial electronics has led to less radiation tolerance and more soft errors in standard commercial electronics [34]. Radiation dosages rise with altitude, making spacecraft and high altitude aircraft more susceptible to radiation effects. Radiation- 
tolerant electronics are very expensive compared to commercial electronics; therefore SAW devices are better candidates for sensors in high altitude and space applications than are devices made from conventional electronics.

\section{CHALLENGES AND ISSUES OF WIRELESS SENSING FOR AEROSPACE VEHICLES}

One of the main challenges for wireless sensors is power [35]. Often batteries cannot be used due to inaccessible locations or exposure to large temperature extremes. Energy harvesting systems that rely on batteries for energy storage are eliminated for the same reasons. Thus, passive wireless sensing systems should be developed because SAW systems can be passive; they do not require batteries. Vibration is another issue. Component failures from the high levels of shock and vibration during operation are not uncommon. Integrated SAW sensors with antennas and proper attachment to structures could enable the devices to survive vibration and mechanical shocks. Pressure variations from vacuum to high pressures should not be an issue for solid state devices like SAWs. For most cases, corona discharge and arcing at low pressures should not pose a problem because the voltages are low. An issue may arise, however: increasing the frequency of the SAW operation will reduce the spacing between the SAW interdigitated fingers, allowing corona discharge and arcing to occur at lower voltages. Since SAW devices are inherently radiation hardened up to 10 MRads, radiation is not a concern. Volume, mass, and power, which are major concerns for most wireless systems, are not an issue for SAW devices. SAW systems can operate over a very wide temperature range; however, temperature variations will cause the output frequency to shift. This is a minor concern, however, because compensation techniques already exist.

RF communication issues pose a significant challenge to implementing SAW systems successfully. Modulation methods must be chosen to allow large numbers of devices to communicate without interference. The bandwidth must be utilized carefully to enable high data rates, while adhering to FCC limitations. Encoding schemes must be developed to allow for efficient operation in noisy environments. The higher frequencies mean smaller antenna sizes and higher data rates. Feature sizes must shrink, too, possibly leading to manufacturing issues. Also, the frequency of operation must follow FCC guidelines. Electromagnetic interference poses a problem for all wireless systems. All wireless electronics must be designed to be pass tests for both electromagnetic compatibility and interference [36].

Certification of wireless sensor networks for flight is another issue that must be addressed. This includes the allocation of frequencies for wireless sensing on aircraft, along with the determination of RF power levels, and FAA acceptance for aircraft use. There is a concern that wireless devices within the cabin may interfere with aircraft antennas located outside the cabin [37]. Personal electronic devices and cell phones have been tested to determine the effects of wireless devices may have on the aircraft avionics. Active RFID tags have been tested for compliance with RTCA/DO160 aircraft emission limits [38]. The tags exceeded the thresholds, but further investigations are needed before the effects from the interference can be understood. The interference from passive tags was not considered a concern, however, because the study focused on RFID tags contained in cargo pallets without an interrogator. Unfortunately, passive SAW sensors would require an active interrogator within the aircraft structure. Because SAW sensors will share the same frequency bands with RFID tags, and some of the same RF modulation techniques, they may exceed the emission thresholds as well. Before any wireless sensor system can be certified for use on aircraft, it will have to be tested in both large and small aircraft.

\section{CONCLUSIONS}

Passive wireless SAW sensor technology offers many opportunities for application to IVHM sensing. NASA applications include acceleration, temperature, pressure, strain, shape, chemical, acoustic emission, ultrasonics, imaging, eddy current, thermography, and terahertz waves. Each of these applications has its own requirements and issues. However, SAW technology has already demonstrated capabilities for sensing and wireless communication that make it worthy of further investigation. Despite current challenges, SAW is an enabling technology that offers benefits that will allow the incorporation of large numbers of IVHM sensors to proliferate on aircraft.

The need for passive wireless SAW sensors has been identified, but NASA does not possess all of the necessary resources to develop them. Thus, NASA encourages partnerships, including those with universities and industry, to aid in the development of the wireless passive sensors for IVHM applications.

\section{REFERENCES}

[1] L. M. Miller, C. Guidi, and T. Krabach, "Space Sensors for Human Investigation of Planetary Surfaces (SpaceSHIPS)," in NASA/JPL 2nd International Conference on Micro/Nanotechnology for Space Applications, Pasadena, CA, 1999, p. 13.

[2] J. Brusey and A. Thorne, "Aero-ID Sensor Integration: Scope of Work, AEROID-CAM-003," University of Cambridge, Cambridge, UK, February 1, 2006, p. 19.

[3] Steering Committee for the Decadal Survey of Civil Aeronautics Aeronautics and Space Engineering "Decadal Survey of Civil Aeronautics: Foundation for the Future (2006)," Washington, D. C., The National Academies Press, 2006.

[4] G. A. Hofbauer, "FMCW Based Readout System Accuracy Enhancement Techniques for Surface Acoustic Wave RFID sensors," in Microwave Symposium, IEEE/MTT-S International, 2007, pp. 575-578.

[5] D. R. Gallagher and D. C. Malocha, "Orthogonal Frequency Coding for Use in Ultra Wide Band Communications and Correlators," in International Frequency Control Symposium and Exposition, 2006 IEEE, 2006, pp. 494-499.

[6] W. E. Bulst, G. Fischerauer, and L. Reindl, "State of the Art in Wireless Sensing with Surface Acoustic Waves," Industrial Electronics, IEEE Transactions on, vol. 48, pp. 265-271, 2001.

[7] L. Reindl, G. Scholl, T. Ostertag, et al., "SAW Devices as Wireless Passive Sensors," in Ultrasonics Symposium, 1996. Proceedings., 1996 IEEE, vol. 1, 1996, pp. 363-367. 
[8] G. Scholl, C. Korden, E. Riha, et al., "SAW-Based Radio Sensor Systems for Short-Range Applications," Microwave Magazine, IEEE, vol. 4, pp. 68-76, 2003.

[9] A. Springer, R. Weigel, A. Pohl, et al., "Wireless Identification and Sensing using Surface Acoustic Wave Devices," Mechatronics, vol. 9, pp. 745-756, 1999.

[10] H. Sato, M. Kawase, and M. Saito, "Temperature Dependence of the Faraday Effect in As-S Glass Fiber," Applied Optics, vol. 24, pp. 23002303, June 1, 1985.

[11] S. Sherrit, X. Q. Bao, Y. Bar-Cohen, et al., "BAW and SAW Sensors for In-Situ Analysis," in Proceedings of the SPIE Smart Structures Conference, San Diego, CA, vol. 5050, 2003, p. 11.

[12] R. White, J. Black, B. Chen, et al., "NCC2-5185: Evaluation of Relative Sensitivity of SAW and Flexural Plate Wave Devices for Atmospheric Sensing," NASA Ames Research Center, NASA/CR-97206768, 19980016026, 1997, p. 10.

[13] G. Cardell, F. Noca, K. Watson, et al., "Micro Weather Station for In Situ Atmospheric Measurements in the Troposphere," in SPIE 15th Annual Conference on Aerosense, Orlando, FL, vol. 4393, 2001.

[14] P. Brown, P. Hartmann, A. Schellhase, et al., "Asset Tracking on the International Space Station Using Global SAW Tag RFID Technology," in Ultrasonics Symposium, 2007. IEEE, 2007, pp. 72-75.

[15] B. A. Childers, M. E. Froggatt, S. G. Allison, et al., "Use of 3000 Bragg Grating Strain Sensors Distributed on Four Eight-Meter Optical Fibers During Static Load Tests of a Composite Structure," Smart Structures and Materials 2001: Industrial and Commercial Applications of Smart Structures Technologies, pp. 133-142, 2001.

[16] J. M. Perotti and A. J. Eckhoff, "Latest Development in Advanced Sensors at Kennedy Space Center (KSC)," in Sensors, 2002. Proceedings of IEEE, vol. 2, 2002, pp. 1728-1733.

[17] S. E. Woodard and B. D. Taylor, "A Wireless Fluid-Level Measurement Technique," Sensors and Actuators A: Physical, vol. 137, pp. 268-278, April 8, 2007.

[18] B. Haowei, M. Atiquzzaman, and D. Lilja, "Wireless Sensor Network for Aircraft Health Monitoring," in Broadband Networks, BroadNets'04, Proceedings, First Conference on, 2004, pp. 748-750.

[19] B. Nickerson and R. Lally, "Development of a Smart Wireless Networkable Sensor for Aircraft Engine Health Management," in Aerospace Conference, IEEE Proceedings, 2001, pp. 3255-3262.

[20] M. Reid, B. Graubard, R. J. Weber, et al., "Wireless Eddy Current Probe for Engine Health Monitoring," in Quantitative Nondestructive Evaluation, Green Bay, WI, vol. 700, 2004, pp. 414-420.

[21] D. L. Simon, G. W. Hunter, T. Guo, et al., "Sensor Needs for Control and Health Management of Intelligent Aircraft Engines," NASA/TM2004-213202, p. 17, August 2004.

[22] A. Kovacs, D. Peroulis, and F. Sadeghi, "Early-Warning Wireless Telemeter for Harsh-Environment Bearings," in Sensors, 2007 IEEE, Atlanta, GA, 2007, pp. 946-949.

[23] H. Trang, R. Patrice, V. Marie-Helene, et al., "TemperatureCompensated Structure For Saw Pressure Sensor In Very High Temperature," in Frequency Control Symposium, Joint with the 21st European Frequency and Time Forum, IEEE, 2007, pp. 40-44.

[24] M. N. Hamidon, V. Skarda, N. M. White, et al., "High-Temperature $434 \mathrm{MHz}$ Surface Acoustic Wave Devices Based on GaPO/sub 4,"
Ultrasonics, Ferroelectrics and Frequency Control, IEEE Transactions on, vol. 53, pp. 2465-2470, 2006.

[25] D. Puccio, D. C. Malocha, N. Saldanha, et al., "SAW Parameters on Ycut Langasite Structured Materials," Ultrasonics, Ferroelectrics and Frequency Control, IEEE Transactions on, vol. 54, pp. 1873-1881, September 2007.

[26] W. H. Prosser, "Development of Structural Health Management Technology for Aerospace Vehicles," NASA LaRC, JANNAF 39th CS/27th APS/21st PSHS/3rd MSS Joint Subcommittee Meeting, 20031216, 2003, p. 9.

[27] T. E. Noll, S. D. Ishmael, B. Henwood, et al., "Technical Findings, Lessons Learned, and Recommendations Resulting from the Helios Prototype Vehicle Mishap," in NATO/RTO AVT-145 Workshop on Design Concepts, Processes and Criteria for UAV Structural Integrity, Florence, Italy, 2007, p. 17.

[28] T. B. Smith, "Development and Ground Testing of Direct Measuring Skin Friction Gages for High Enthalpy Supersonic Flight Tests," Dissertation, Virginia Polytechnic Institute and State University, Blacksburg, VA, Setpember 10, 2001.

[29] G. W. Hunter, P. G. Neudeck, C. C. Liu, et al., "Development of Chemical Sensor Arrays for Harsh Environments and Aerospace Applications," in Sensors, Proceedings of IEEE, vol. 2, June 12-14, 2002, pp. 1126-1133.

[30] J. A. Thiele and M. Pereira da Cunha, "Dual Configuration High Temperature Hydrogen Sensor on LGS SAW Devices," in Ultrasonics Symposium, 2004 IEEE, vol. 2, 2004, pp. 809-812.

[31] W. T. Yost, K. E. Cramer, and D. F. Perey, "Characterization of Effluents Given Off by Wiring Insulation," Review of Progress in Quantitative Nondestructive Evaluation, vol. 23 B, pp. 1155-1161, 2003.

[32] B. H. Fisher and D. C. Malocha, "Cryogenic Liquid Sensing Using SAW Devices," in Frequency Control Symposium, Joint with European Frequency and Time Forum. IEEE International, 2007, pp. 505-510.

[33] W. J. Stapor, J. H. Hines, and D. H. Wilson, "Ionizing Space Radiation Effects on Surface Acoustic Wave Resonators," Nuclear Science, IEEE Transactions on, vol. 38, pp. 1329-1335, 1991.

[34] D. G. Mavis and P. H. Eaton, "Soft Error Rate Mitigation Techniques for Modern Microcircuits," in Reliability Physics Symposium Proceedings, 40th Annual, 2002, pp. 216-225.

[35] S. Roundy, D. Steingart, L. Frechette, et al., "Power Sources for Wireless Sensor Networks," in Wireless Sensor Networks, First European Workshopt, EWSAN, Berlin, Germany, vol. 2920, January 19-21, 2004, pp. 1-17.

[36] T. Vladimirova, C. P. Bridges, G. Prassinos, et al., "Characterising Wireless Sensor Motes for Space Applications," in Adaptive Hardware and Systems, Second NASA/ESA Conference on, 2007, pp. 43-50.

[37] T. X. Nguyen, S. V. Koppen, J. J. Ely, et al., "Small Aircraft RF Interference Path Loss Measurements," NASA Langley Research Center, Hampton, VA, NASA/TP-2007-214891, August, 2007, p. 121.

[38] T. X. Nguyen, J. J. Ely, R. A. Williams, et al., "RFID Transponders' Radio Frequency Emissions in Aircraft Communication and Navigation Radio Bands," NASA Langley Research Center, Hampton, VA, NASA/TP-2006-214295, March, 2006, p. 85. 